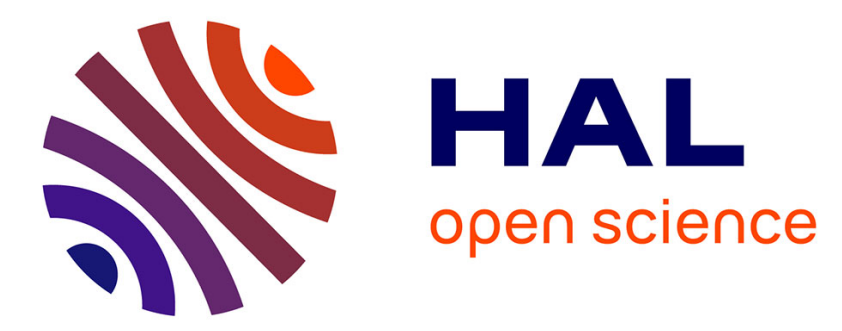

\title{
Hybrid Nonovershooting Set-Point Pressure Regulation for a Wet Clutch
}

Matteo Cocetti, Silvia Donnarumma, Luca de Pascali, Matteo Ragni, Francesco Biral, Fabrizio Panizzolo, Pier Paolo Rinaldi, Alex Sassaro, Luca Zaccarian

\section{To cite this version:}

Matteo Cocetti, Silvia Donnarumma, Luca de Pascali, Matteo Ragni, Francesco Biral, et al.. Hybrid Nonovershooting Set-Point Pressure Regulation for a Wet Clutch. IEEE/ASME Transactions on Mechatronics, 2020, 25 (3), pp.1276-1287. 10.1109/TMECH.2020.2973382 . hal-03029043

\section{HAL Id: hal-03029043 https://hal.science/hal-03029043}

Submitted on 1 Dec 2020

HAL is a multi-disciplinary open access archive for the deposit and dissemination of scientific research documents, whether they are published or not. The documents may come from teaching and research institutions in France or abroad, or from public or private research centers.
L'archive ouverte pluridisciplinaire HAL, est destinée au dépôt et à la diffusion de documents scientifiques de niveau recherche, publiés ou non, émanant des établissements d'enseignement et de recherche français ou étrangers, des laboratoires publics ou privés. 


\title{
Hybrid non-overshooting set-point pressure regulation for a wet clutch
}

\author{
Matteo Cocetti, Silvia Donnarumma, Luca De Pascali, Matteo Ragni, Francesco Biral, Fabrizio Panizzolo, \\ Pier Paolo Rinaldi, Alex Sassaro, and Luca Zaccarian, Fellow, IEEE
}

\begin{abstract}
We propose a control oriented Wiener model for wet-clutches in filled conditions and we discuss the associated identification technique. We design a novel hybrid controller, which ensures zero steady-state error and a fast non-overshooting response. We show that the controller parameters can be conveniently obtained by solving a set of linear matrix inequalities. Finally, we test the proposed control strategy on the hydromechanical variable transmission developed by Dana-Rexroth Transmission Systems. The experiments show good performance and robustness with respect to modeling errors and noise.
\end{abstract}

Index Terms-Hybrid/reset control, overshoot, switching control, linear matrix inequalities, wet-clutch, pressure control.

\section{INTRODUCTION}

The main goals for the next generation of transmission systems are an improved fuel economy and a better productivity. Toward these goals the automotive industry has developed numerous solutions, such as automatic and dual clutch transmissions, which can automatically shift among different drive ranges, thus improving both vehicle drivability and fuel economy. These technologies are now mature enough to be employed also in the off-highway, agricultural and working machines industry. However, it is particularly challenging to adapt these technologies for the off-highway market due to the rather different requirements. For example, mechanical continuous variable transmissions are very efficient, but they operate in a too narrow power range. This problem can be partially solved using hydrostatic transmissions, but their efficiency is low. Ideally, the best solution should combine the efficiency of the mechanical continuous variable transmissions and the power range of a hydrostatic transmission. This marriage between the hydrostatic and the mechanical world is the hydro-mechanical variable transmission (HVT) that has been introduced by Dana-Rexroth Transmission Systems (DRTS) in [1] and references therein.

S. Donnarumma is with the department of Marine Engineering and Naval Architecture, University of Genoa, 1 Via Montallegro, Genova, IT, 16145. Email: donnarumma@dime.unige.it.

M. Cocetti, L. De Pascali, M. Ragni, F. Biral and L. Zaccarian are with the Department of Industrial Engineering, 9 Sommarive, University of Trento, Trento, IT, 38123. Email: (matteo.cocetti, luca.depascali, matteo.ragni, francesco.biral, luca.zaccarian) dunitn.it. L. Zaccarian is also with LAASCNRS, Université de Toulouse, CNRS, Toulouse, France.

F. Panizzolo, P. P. Rinaldi and A. Sassaro are with Dana Rexroth Transmission Systems S.r.l, Località Linfano, IT, 38062 Arco (TN). Email: (alex.sassaro, pierpaolo.rinaldi, Fabrizio.Panizzolo) (danarexroth.com.

Research supported by grant PowerLyap funded by CaRiTRo, by Dana Rexroth Transmission Systems S.r.l., and in part by ANR via grant HANDY, number ANR-18-CE40-0010.

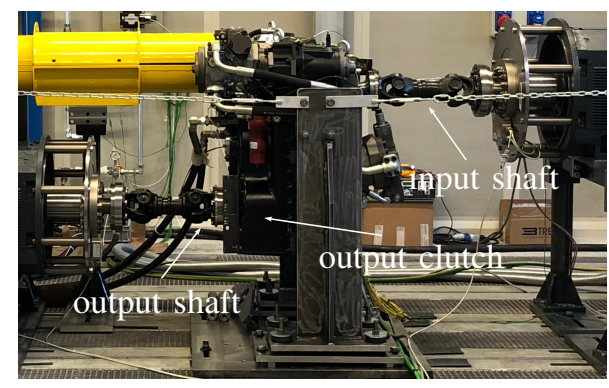

Fig. 1. The hydro-mechanical variable transmission.

In the HVT architecture, see Figure 1, the engine power is split into two different pathways: the first one is mechanical and is highly efficient, while the second one is hydrostatic and preserves the continuous variable transmission characteristic. The powers coming from each pathway are then combined by means of a planetary gear. Moreover, in order to extend the range of possible speeds, the HVT can also shift among several drive ranges. This shift is performed by coordinating several wet-clutches, suitably actuated by electro-proportional valves. Ideally, during a clutch shift, the transmitted torque should remain constant to ensure a comfortable and smooth transition.

Clutch control is an active area of research, and numerous control techniques have been proposed in the literature. In [2], [3] the authors propose a combination of differential flatness and feedback linearization, while in [4] a robust sliding-mode controller is presented. These approaches are rather elegant, but they are usually hard to implement in a digital form due to discretization issues or poorly known dynamics. This is especially true in our setup, where the actuation system of the HVT is difficult to identify precisely, due to non-linear effects, hysteresis and dependence on the oil temperature. Consequently, complex controllers may fail to work properly. Moreover, in contrast to [2] and [3], we do not assume the knowledge of the piston position, because for wet clutches it is technologically very hard to place sensors inside a chamber with a rotating shaft soaked into oil. For this reason, the only available measurement for feedback is the oil pressure. A similar setup has been already considered in [5], where the authors propose a linear model for a twin-clutch transmission and they design a pressure feedback controller inducing a desirable clutch shift.

In this work we follow a similar spirit, but compared to [5], we consider a more complete model and we propose a novel hybrid controller. The main differences are highlighted below. 
First, in our setup the clutch shows a non-constant DC gain and a small variable delay in the actuation. We include these effects by adding a non-linear invertible map and considering a non-minimum phase approximation of the delay. The resulting model is the cascade of a non-minimum phase single input single output linear time-invariant system and an invertible non-linear output map, a so-called Wiener model.

Second, since a large amount of power is transmitted by the HVT, the closed-loop specifications are tight and somehow conflicting. The step response of the pressure in the chamber must be fast, non-overshooting, and with zero steady-state error. However, it has been shown in [6] (see also [7]) that linear linear controllers with integral action (to ensure zero steady-state error) combined with a sufficiently slow rise time (that is, some reasonable bandwidth specification), necessarily leads to an overshoot. To overcome this limitation, we propose a novel hybrid controller that combines a switching and a resetting mechanism, thus providing the desired response. The controller synthesis is conveniently formulated as a linear matrix inequality (LMI) problem. A preliminary version of this work has been presented in [8]. Here we provide an improved LMI-formulation of the non-overshooting design, and we introduce the hybrid controller with integral action which was absent in [8]. Moreover, we study the robustness properties of the proposed solution considering constant (or slowly varying) disturbances acting on the model. Finally, we provide new and more convincing experimental results, showing the advantages of the proposed controller.

The paper is organized as follows: in Section I we introduce and we motivate the work. In Section II we introduce the experimental setup and the closed-loop goals. In Section III we propose a control-oriented model for wet clutches and the related identification technique. In Section IV we present the novel hybrid controller. In Section V we propose an LMIbased tuning procedure for the proposed controller. Experimental tests are presented and discussed in Section VI. Finally, conclusions and remarks are offered in Section VII.

For reasons of confidentiality all the units of measure are normalized and the technological details are omitted. This does not affect the contribution of the proposed control design which is fully parametric and has been validated on five different clutches, differing in mechanical and hydraulic properties. Indeed, our construction can be implemented on any exponentially stable second order plant, thereby making our technique broadly applicable.

\section{SETUP DESCRIPTION AND GOALS}

In the HVT architecture, wet-clutches are actuated by the hydraulic circuit sketched in Figure 2. The current in the valve regulates the oil pressure inside the clutch chamber, and controls in an indirect way the piston movement. Through this mechanism we can open and close the clutch, and by increasing or decreasing the contact force we modulate the transmitted torque. The standard actuation sequence is as follows: first, the current in the valve increases opening the orifice between the clutch chamber and the supply high pressure line. The oil flows pushing the clutch piston towards the end of

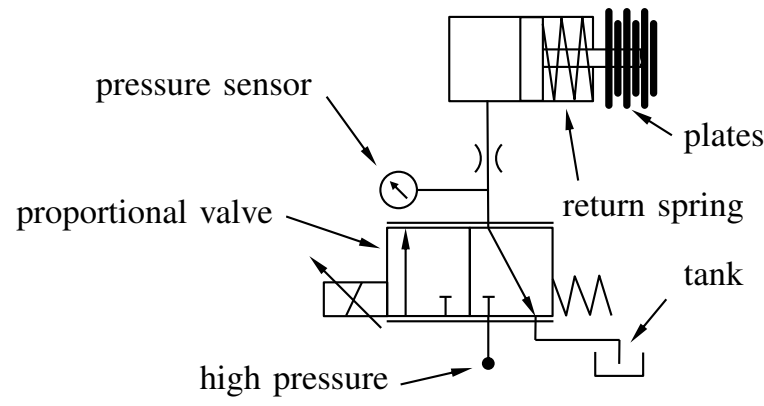

Fig. 2. Hydraulic actuation scheme.

the stroke, causing the transmission of a small torque due to drag effects. This phase is known as filling phase, and it is usually performed in an open-loop fashion. During the filling phase, the pressure measurements are not reliable due to the pressure gradient associated to the flow. Secondly, when the piston reaches the end of the stroke, the gap between the clutch plates is zero and the flow stops. At this pressure, known as (mechanical) kiss point [9], the piston remains in equilibrium, thanks to the balancing between the oil pressure and the force exerted by the spring. Thirdly, after the kiss point, the plates push against each other and transmit a considerable amount of torque. This operating condition is known as modulation range, and is characterized by an affine relationship between the oil pressure and the transferred torque.

In order to obtain a smooth clutch shift, the filling phase, the kiss point, and the modulation range, need to be properly controlled. This translates into three key ingredients: 1) a correct timing and synchronization of the clutches, 2) a well prepared on-coming clutch (filled with oil and ready to engage), 3) a precise oil pressure control in the modulation range. In this paper we focus on the aspect of controlling the oil pressure in the modulation range. Because in the modulation range there is an affine relationship between the oil pressure and the transmitted torque, the task of controlling the torque is indeed equivalent to controlling the pressure. Here we assume that the electrical dynamics of the valve are negligible, i.e., much faster than the mechanical ones, because a high performance current control has been implemented [10].

Even under the above simplifying assumptions, precisely controlling the oil pressure is a challenging task. The nonlinear interaction between the valve and the oil, and the viscosity that changes with the temperature, make the task non-trivial.

For this application it is also fundamental that the closedloop satisfies the following tight performance goals, whose motivations are discussed next.

Problem 1 (Control problem). Design an oil pressure controller for the HVT such that the closed-loop response satisfies the following conditions:

1) Nominal non-overshooting step response.

2) Very small rise time.

3) Zero steady-state error for constant reference signals.

The precise value of the rise time is not specified for confidentiality reasons. These goals are all equally important to achieve a smooth clutch-shift because items 2) and 3) are key to ensuring that the driver does not experience any 
perturbation during the shift operation. Moreover, among all, the non-overshooting constraint is especially critical. Because, overshoot in the pressure response causes an excess of dissipated power that could burn the clutch friction discs.

Problem 1 is difficult because requirements 1) and 3) are conflicting, and 2) imposes to use an aggressive controller.

In addition to the goals defined in Problem 1, it is desirable that the controller is robust w.r.t. 1) small delays in the loop, 2) possible slow unmodeled dynamics, 3) variations of the oil temperature and clutch aging. Moreover, due to the limited electronic hardware in the automotive industry, the proposed controller must be "easy enough" to be implemented with a limited computational power.

\section{MODELING AND IDENTIFICATION}

\section{A. Model selection}

In this section we discuss a control-oriented model for wet clutches in the modulation range. The input is the current in the valve, the output is the oil pressure inside the clutch chamber.

We performed a series of experiments, using a staircase-like input current, as shown in Figure 3. The response at the first step differs from the others due to the presence of flowing oil (the clutch is still in the filling phase). When the clutch reaches the modulation range, the oscillating behavior resembles a second-order linear system with complex poles, however a closer look reveals some nonlinear effects. First, the DC gain is not constant, secondly there is a small variable input-output delay, probably associated to a non-constant computational time. We capture the non-constant DC gain through a static output nonlinearity $\phi$, for which we assume the following.

Assumption 1. The output non-linearity $\phi$ is a continuous strictly increasing function on $\mathbb{R}_{\geq 0}:=[0,+\infty)$.

For the input delay $d$, we introduced a non-minimum phase behavior in the response, which approximates the delay by way of a small undershoot. This is not surprising since the first-order Padé approximation of delay $d$ yields,

$$
e^{-i \omega d} \approx \frac{1-i \omega d / 2}{1+i \omega d / 2} \quad \forall \omega \ll 1 / d,
$$

where $i$ is the imaginary unit and $\omega \in \mathbb{R}$ the frequency. This approximation shows that for a large range of frequencies, $(1 / d$ is large), a small time delay is equivalent to a zero in the open right-half plane plus a pole in the open left-half. We can disregard the pole, because it is much faster than the clutch dynamics, and we keep the zero.

With slight abuse of notation, we mix time and Laplace domain. Given a signal $u(\cdot)$ defined for $t \in \mathbb{R}_{\geq 0}$, we denote by $u(t) \in \mathbb{R}$ the value at time $t$, and by $u(s) \in \mathbb{C}$ the value of its Laplace transform at $s \in \mathbb{C}$. Based on the considerations above, we propose a Wiener model [11], of the following form

$$
\begin{aligned}
y(s) & :=\left(\frac{1+a_{3} s}{1+a_{1} s+a_{2} s^{2}}\right) u(s)=H(s) u(s) \\
z(t) & :=\phi(y(t)),
\end{aligned}
$$

where $u \in \mathbb{R}$ is the input, $z \in \mathbb{R}$ is the measured output, $y \in \mathbb{R}$ is an equivalent "linear output" and $\phi: \mathbb{R}_{\geq 0} \rightarrow \mathbb{R}_{\geq 0}$ an invertible nonlinear map.

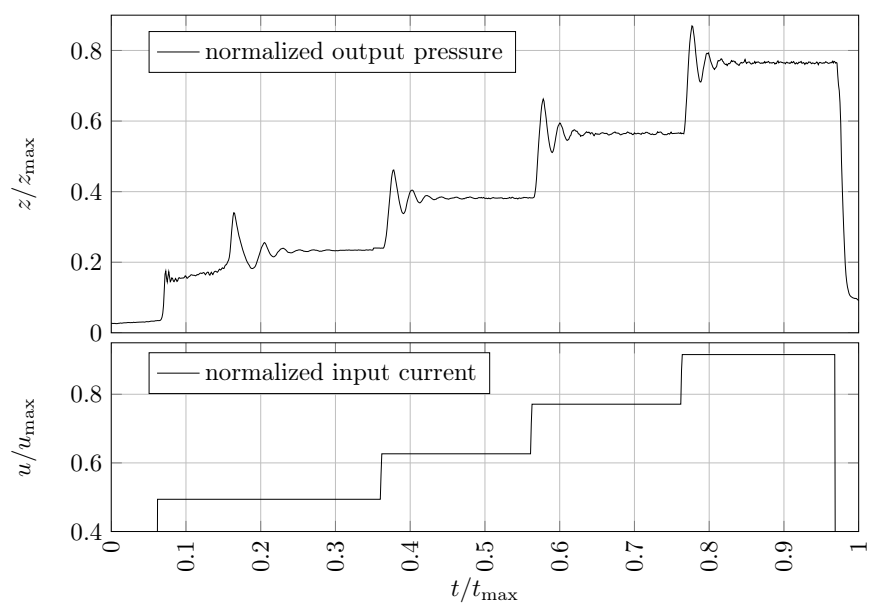

Fig. 3. Experimental pressure response to a staircase input current. $z \in \mathbb{R}$ is the pressure and $u \in \mathbb{R}$ is the input current, while $z_{\max }, u_{\max }$ and $t_{\max }$ are normalization factors.

The transfer function $H(s) \in \mathbb{C}$ is strictly proper and nonminimum phase due to the delay approximation in (1).

Remark 1. Without loss of generality we assume that the DC gain of (2a) is unitary, $\lim _{s \rightarrow 0} H(s)=1$. Indeed, a different DC gain can be easily absorbed into the nonlinearity $\phi$.

\section{B. Model identification}

Identification of general Hammerstein-Wiener models is a complex topic, but in this paper thanks to the invertibility of $\phi$ (see Assumption 1) the identification procedure boils down to a simple two-step procedure. First we identify $\phi$ considering a large set of steady-state input-output pairs $\left\{\left(u^{i}, z^{i}\right)\right\}_{i=1}^{N} \subset$ $\mathbb{R}^{2}$ (where $N \gg 1$ ). Then we identify the remaining linear dynamics using standard tools. Since the transfer function (2a) has unitary DC gain, the pairs $\left(u^{i}, z^{i}\right)$ are related through $\phi$, and the identification of the output nonlinearity reduces to an interpolation problem. The second order polynomial

$$
z^{i}=\phi\left(u^{i}\right):=p_{0}+p_{1} u^{i}+p_{2}\left(u^{i}\right)^{2}+\epsilon^{i}, \quad i=1, \ldots, N,
$$

provides a good fitting, see Figure 4. The coefficients $p_{0}, p_{1}, p_{2} \in \mathbb{R}$ can be easily obtained by solving a least squares problem. Once $\phi$ is known, we estimate the coefficients $a:=$ $\left(a_{1}, a_{2}, a_{3}\right) \in \mathbb{R}^{3}$ virtually accessing the linear output $y$ as $y=\phi^{-1}(z)$ and using any standard identification techniques for linear systems, see for example [11]. Notice that general quadratic functions of the form (3) are not globally invertible, as required in Assumption 1, however (3) is experimentally invertible over $\mathbb{R}_{\geq 0}$ for the identified set of parameters.

\section{Balanced realization}

In Section V we will describe an LMI-based procedure for the controller synthesis. Because LMIs are naturally formulated in state space, we consider a minimal realization of (2a) as follows

$$
\mathcal{H}_{\mathrm{p}}:=\left\{\begin{aligned}
\dot{x}_{\mathrm{p}} & =A x_{\mathrm{p}}+B u+G w \\
y & =C x_{\mathrm{p}}+Q w \\
z & =\phi(y)
\end{aligned}\right.
$$




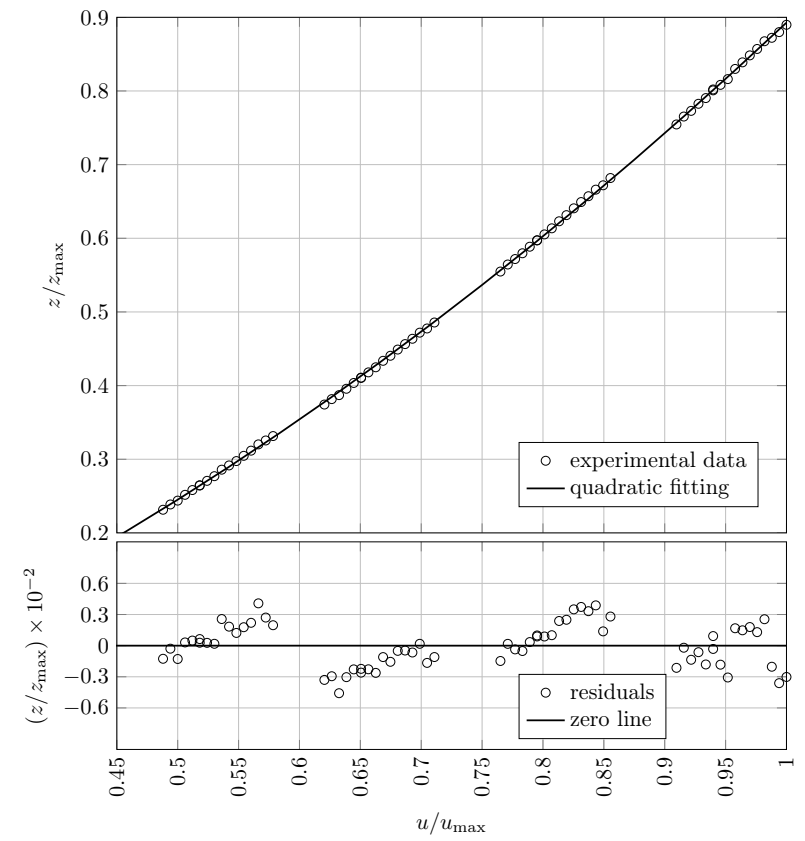

Fig. 4. Interpolation of the steady-state pairs (top) and residuals (bottom) normalized with respect to the maximum pressure $z^{\max }$.

where $x_{\mathrm{p}} \in \mathbb{R}^{2}$ is the state, and $w \in \mathbb{R}$ is a disturbance. Matrices $G$ and $Q$ cannot be directly obtained from (2a), but can be freely designed to "shape" the effect of the disturbance $w$ on the measurement and the state. For (4), we used a balanced realization [12], which guarantees that equally "important" states have similar magnitude. This improves numerical stability and makes the LMIs in Section IV better conditioned from a numerical viewpoint. Moreover, according to Remark 1, we assume that (4) satisfies the following:

Assumption 2. Matrix $A$ is Hurwitz, the triple $(C, A, B)$ is minimal, balanced and satisfies $-C A^{-1} B=1$.

According to the goal of set-point regulation defined in Problem 1, we restrict the class of possible reference and disturbance signals to constant ones.

Assumption 3. The signals $r$ and $w$ are constant.

Remark 2. Assumptions 2, 3 are not restrictive for the experimental system under consideration, and the presence of the output nonlinearity $\phi$ adds no conceptual difficulties, because thanks to Assumption 1 we can always access $y$ through $\phi^{-1}$. It is important to stress that an imperfect knowledge of the output non-linearity $\phi$ produces a constant steady-state error that can be thought of as part of $w$. Therefore, from this point over, and especially in Section IV, we consider to have full access to signal $y$.

\section{HYBRID CONTROLLER}

In this section we propose a novel hybrid control scheme solving Problem 1 by switching among two different modes. The first mode corresponds to a "transient mode" that shapes the transient providing a fast non-overshooting step response. The second mode is a "steady-state mode" that activates an integral action able to zeroing out the steady-state error. We emphasize that a linear controller simultaneously satisfying the requirements defined in Problem 1 may not exists. Indeed the combined action of the non-minimum phase zero and of the integrator (necessary to ensure zero steady-state error) considerably reduces the phase margin of the open loop, thus making the design of a non-overshooting controller a challenging task. Intrinsic limitations of linear controllers are a well known subject in the literature [6], [7], and for this reason we decided to adopt a hybrid controller with a switching mechanism. Switching among different controllers has already been recognized as an effective way to obtain superior performance [13]. This technique is also popular in the industry, where it is widely used in the context of gain scheduling. However, it is well-known that the transients caused by switching may result in instability of the closed-loop. Motivated by this fact, many researchers proposed stability conditions for switching controllers, see for example [14], [15], just to cite a few.

To overcome the problem of destabilizing transients we equip our controller with a bumpless filter [16], and a properly designed reset rule. The interplay between the bumpless dynamics and the resets guarantees stability under arbitrarily switching. This strong property leaves complete freedom in the design of the high-level switching logic.

A graphical representation of model (2) in feedback interconnection with the hybrid controller is shown in Figure 5. We may notice that controller $\mathcal{H}_{\mathrm{c}}$ requires two inputs: the "tracking error" $e \in \mathbb{R}$ and the "feed-forward" control $u_{\mathrm{ff}} \in \mathbb{R}$. The "feed-forward" control $u_{\mathrm{ff}}$ is obtained by inverting the Wiener non-linearity $\phi$ as follows

$$
u_{\mathrm{ff}}:=\phi^{-1}(r)
$$

where $r$ is the reference signal for $z$. Because by Assumption 2 the DC gain of (2a) is unitary, $u_{\mathrm{ff}}$ can be interpreted also as a reference signal for $y$ and it makes sense to define the tracking error $e$ as

$$
e:=y-u_{\mathrm{ff}} .
$$

We notice that thanks to Assumption 1, the non-overshooting requirement for $(z-r)$ can be equivalently considered on $e$. The proposed hybrid controller $\mathcal{H}_{\mathrm{c}}$ has the following structure:

$$
\mathcal{H}_{\mathrm{c}}:=\left\{\begin{array}{c}
\dot{x}_{\mathrm{c}}=A_{\mathrm{c}}(q) x_{\mathrm{c}}+B_{\mathrm{c}}(q) u_{\mathrm{c}} \\
x_{\mathrm{c}}^{+}=E_{\mathrm{c}}(q) x_{\mathrm{c}}+F_{\mathrm{c}}(q) u_{\mathrm{c}}
\end{array}\right.
$$

where $x_{\mathrm{c}} \in \mathbb{R}^{6}$ is the controller state, $u_{\mathrm{c}}:=\left(e, u_{\mathrm{ff}}\right) \in \mathbb{R}^{2}$ is the controller input, and $q \in \mathcal{Q}:=\{1,2\}$ is a logic variable selecting what mode is currently active. Moreover, controller (8) is interconnected to (4) through the following output equation:

$$
u=C_{\mathrm{c}}(q) x_{\mathrm{c}}+D_{\mathrm{c}}(q) u_{\mathrm{c}} .
$$

The logic value $q=1$ is associated to the "transient mode", while $q=2$ is associated to the "steady-state mode". Explicit expressions for the matrices in (8), (9) for each mode are reported in (5).

Remark 3. Notice that (8) includes a reset mechanism, namely a discontinuity of the controller state $x_{\mathrm{c}}$. This discontinuity is imposed when the logic variable $q$ toggles between values 1 


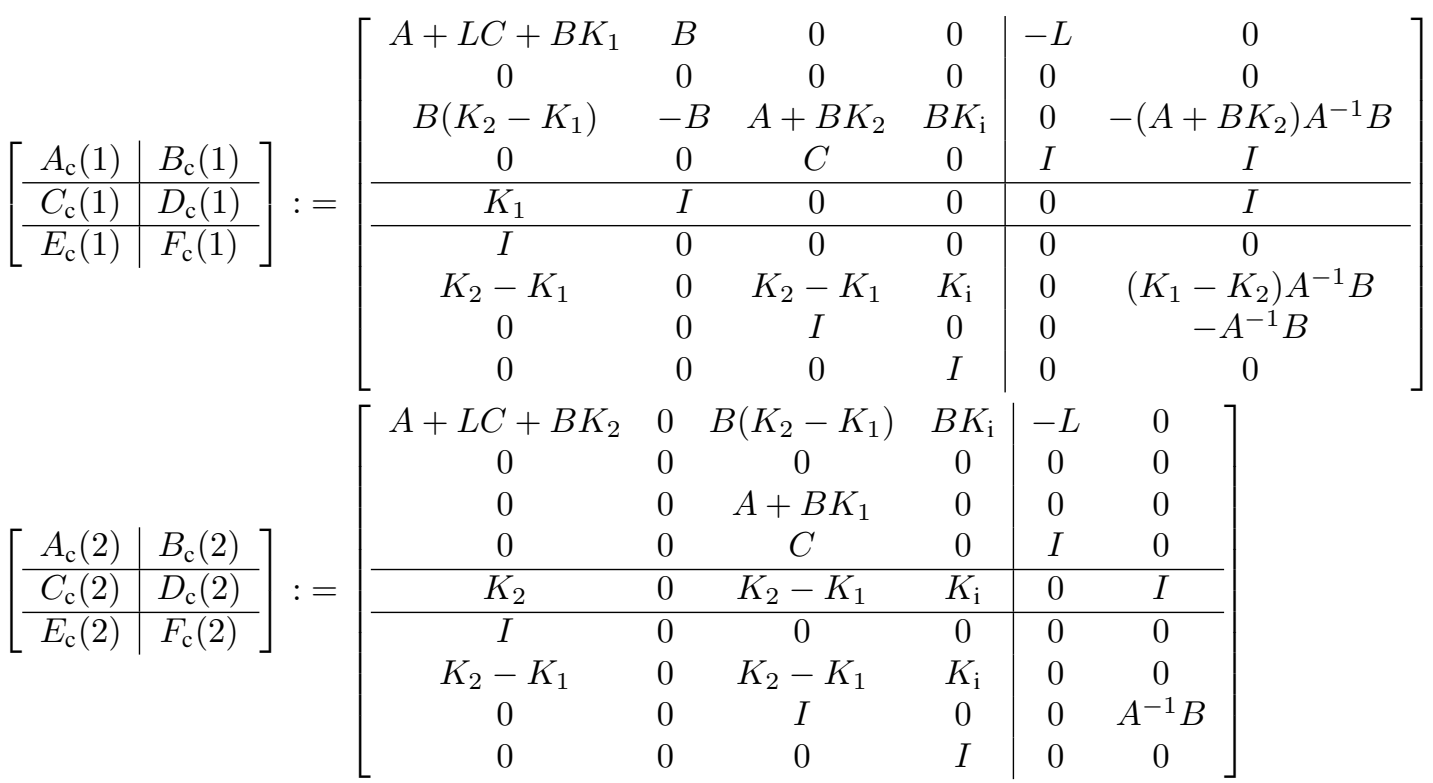

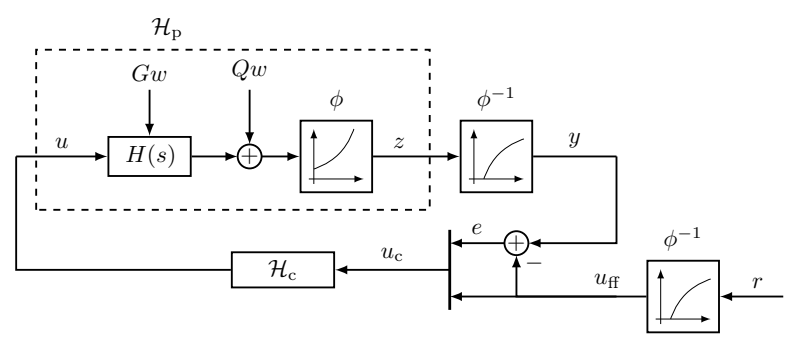

Fig. 5. Block diagram of the model-controller loop. Please notice the presence of $\phi^{-1}$ and the error feedback hybrid controller.

and 2. The motivation for this reset mechanism will become apparent in the proof of Theorem 1 where we will include the logic variable $q$ as a part of the state, so that $x_{\mathrm{c}}$ and $q$ are updated synchronously during jumps.

Although the structure of Equation (5) may seem complicated, there is a rather intuitive motivation for each block. Looking at (5) we can split the controller state into 4 different sub-states as follows $x_{\mathrm{c}}=\left(x_{\mathrm{c} 1}, x_{\mathrm{c} 2}, x_{\mathrm{c} 3}, x_{\mathrm{c} 4}\right) \in$ $\mathbb{R}^{2} \times \mathbb{R} \times \mathbb{R}^{2} \times \mathbb{R}$, where we used the shorthand notation $\left(x_{1}, x_{2}\right):=\left[x_{1}^{\top}, x_{2}^{\top}\right]^{\top}$. The individual role of each component of the controller state is clarified below:

1) $x_{\mathrm{c} 1}$ is associated to a Luenberger observer that provides an estimate of the state $x_{\mathrm{p}}$ in (4);

2) $x_{\mathrm{c} 2}$ introduces a constant input bias that plays the role of an integral action updated only during jumps;

3) $x_{\mathrm{c} 3}$ corresponds to a bumpless filter, inspired by [16], which guarantees stability under a large class of switching signals;

4) $x_{\mathrm{c} 4}$ implements the integral action of the steady-state controller $(q=2)$.

Finally the reset mechanism for (8) is specified by the matrices $E_{\mathrm{c}}(q)$ and $F_{\mathrm{c}}(q)$. The resets and the switches are designed to keep the solutions well behaved across jumps and, in particular, it ensures that the feed-forward signal $u_{\mathrm{ff}}$ enters only in the sub-block of the controller relative to the active mode. This fact ensures that $q$ can switch arbitrarily fast between the two modes without destabilizing the closed-loop. Moreover, when switching from $q=2$ to $q^{+}=1$, the reset exploits the information provided by $x_{\mathrm{c} 3}, x_{\mathrm{c} 4}$ to update the bias generated through $x_{c 2}$ and asymptotically recovers zero steady-state error also in transient mode $(q=1)$.

The above properties are more evident when looking at the interconnection between (4) and (8) through (9) and (7), in a different set of coordinates. Thanks to Assumption 3 we have that $\dot{u}_{\mathrm{ff}}=0$ and we can consider the change of coordinates

$$
x:=T(q)\left(x_{\mathrm{p}}, x_{\mathrm{c}}\right)+U(q) u_{\mathrm{ff}},
$$

where matrices $T(q) \in \mathbb{R}^{8 \times 8}$ and $U(q) \in \mathbb{R}^{8 \times 1}$ are reported below

$$
\begin{aligned}
& (T(q), U(q)):= \\
& {\left[\begin{array}{ccccc|c}
(2-q) I & 0 & 0 & (1-q) I & 0 & (2-q) A^{-1} B \\
0 & 0 & I & 0 & 0 & 0 \\
I & 0 & 0 & I & 0 & (q-1) A^{-1} B \\
0 & 0 & 0 & 0 & I & 0 \\
-I & I & 0 & 0 & 0 & -A^{-1} B
\end{array}\right] .}
\end{aligned}
$$

Notice that $T(q), U(q)$ are full rank for all $q \in \mathcal{Q}$ so that (10) is always well-defined.

Thanks to (10) the closed-loop takes the simplified expression

$$
\mathcal{H}:=\left\{\begin{aligned}
\dot{x} & =A_{\mathrm{cl}}(q) x+G_{\mathrm{cl}}(q) w \\
x^{+} & =E_{\mathrm{cl}}(q) x,
\end{aligned}\right.
$$

where $A_{\mathrm{cl}}(q) \in \mathbb{R}^{8 \times 8}, G_{\mathrm{cl}}(q) \in \mathbb{R}^{8 \times 1}$, and $E_{\mathrm{cl}}(q) \in \mathbb{R}^{8 \times 8}$ are reported in Equation (12). Similarly, the tracking error $e$ yields

$$
e=C_{\mathrm{cl}}(q) x+D_{\mathrm{cl}} w,
$$

where again $C_{\mathrm{cl}}(q) \in \mathbb{R}^{1 \times 8}, D_{\mathrm{cl}} \in \mathbb{R}^{1 \times 1}$ are defined in Equation (14). It is worth to notice that (14) has a desirable cascade structure and thus a necessary condition for stability is to ensure that the diagonal sub-blocks

$$
\left[A+B K_{1}\right], \quad\left[\begin{array}{cc}
A+B K_{2} & B K_{\mathrm{i}} \\
C & 0
\end{array}\right], \quad[A+L C]
$$


are Hurwitz. This is not restrictive in our setup, since the triple $(C, A, B)$ is minimal from Assumption 2. Then it is always possible to find a set of parameters $K_{1}, K_{2}, K_{\mathrm{i}}, L$ such that the matrices in (13) are Hurwitz. We illustrate a possible design procedure in sections V-A, V-B and V-C. Finally, to study the stability properties of (11) we assume that the switching signal $q$ satisfy a mild dwell-time and reverse dwell-time condition. Denoting by $t_{j}, j \in \mathbb{N}_{>1}$ the toggle times, i.e. the times when the logic variable $q$ changes from $q=1$ to $q^{+}=2$ or viceversa, we assume the following.

Assumption 4. There exist two positive numbers $\tau_{\min }, \tau_{\max } \in$ $\mathbb{R}_{>0}$, such that $\tau_{\text {min }} \leq\left|t_{j+1}-t_{j}\right| \leq \tau_{\max }, \forall j \in \mathbb{N}_{\geq 1}$.

Remark 4. We remark that no constraints on $\tau_{\min }$ and $\tau_{\max }$ are imposed, and that these values are not used in the controller synthesis presented in Section V. Thus Assumption 4 virtually allows for all the possible switching sequences that are relevant from a practical viewpoint.

What follows is the main result of the paper.

Theorem 1 (Main result). Assume that the gains $K_{1}, K_{2}$, $K_{\mathrm{i}}, L$ have been designed so that the matrices in (13) are Hurwitz. If Assumptions 1, 2, 3, and 4 hold true, then (11) has the following properties:

1) For $w=0$ the origin of (11) is uniformly globally exponentially stable for all switching sequences.

2) For any constant $w$ the solutions to (11) are globally bounded and ensure asymptotic convergence to zero of $e$.

Theorem 1 provides a strong and desirable result because it establishes asymptotic tracking and exponential convergence for any switching signal $q$ satisfying the mild requirements in Assumption 4.

Proof. To properly describe the class of switching signals defined in Assumption 4 we develop a hybrid representation for (11), which includes $q$ and a timer $\tau$ as part of the state. We use the hybrid systems framework presented in [17]. The following representation is inspired by the construction in [18, page 747] and can be shown to generate all and no more than the switching sequences characterized in Assumption 4, see [18, Prop. 1.1].

The resulting hybrid system has the following structure:

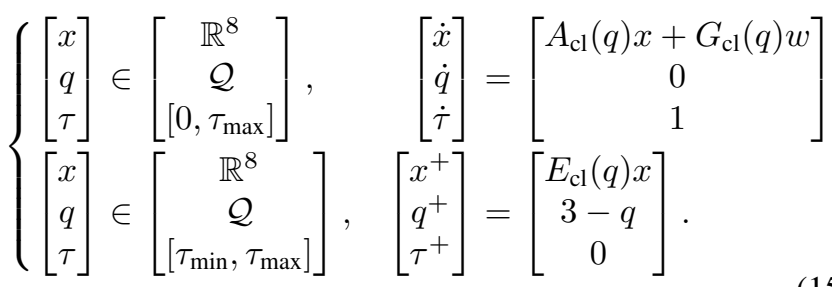

Using the above representation, we proceed to proving the two items of the theorem.

Proof of item 1). Because we are interested in studying the stability properties of the origin of (11) and in (15) we introduced the additional states $(q, \tau)$ we consider the compact attractor $\mathcal{A}:=\{0\} \times \mathcal{Q} \times\left[0, \tau_{\max }\right]$ and the distance function $|x|_{\mathcal{A}}:=\inf _{a \in \mathcal{A}}(|x-a|)$, so that $|(x, q, \tau)|_{\mathcal{A}}=$ $|x|$, and we equivalently study the stability property of $\mathcal{A}$. According to the representation in (14) we split the state $x=\left(x_{1}, \ldots, x_{5}\right) \in \mathbb{R} \times \mathbb{R}^{2} \times \mathbb{R}^{2} \times \mathbb{R} \times \mathbb{R}^{2}$ and we apply the recursive reduction theorem reported in [19, Thm 4] with the following sets: $\Gamma_{4}:=\left\{(x, q, \tau): x_{5}=0\right\}, \Gamma_{3}:=\{(x, q, \tau) \in$ $\left.\Gamma_{4}:\left(x_{3}, x_{4}\right)=0\right\}, \Gamma_{2}:=\left\{(x, q, \tau) \in \Gamma_{3}: x_{2}=0\right\}$, $\Gamma_{1}:=\left\{(x, q, \tau) \in \Gamma_{2}: x_{1}=0\right\}=\mathcal{A}$. To apply [19, Thm 4] we first observe that for the case $w=0$ (addressed in item 1), the following holds:

1) $\Gamma_{4}$ is asymptotically stable because the flow dynamics of $x_{5}$ are independent of the other states and governed by a Hurwitz linear time-invariant flow equation $A+L C$, see (13), while the jump equation is the identity. Asymptotic stability of $\Gamma_{4}$ then follows from the persistent flowing results of [17, Prop. 3.27] ensured by the fact that jumps are inhibited in (15) until $\tau \geq \tau_{\text {min }}$. The same argument can be repeated to prove that $\Gamma_{i}$ is asymptotically stable relative to $\Gamma_{i+1}$, for $i=3,1$, because both $\left[\begin{array}{cc}A+B K_{2} & B K_{\mathrm{i}} \\ C & 0\end{array}\right]$ and $A+B K_{1}$ are Hurwitz by assumption, see (13). To prove that $\Gamma_{2}$ is asymptotically stable relative to $\Gamma_{3}$, we first project the dynamics on a reduced state space where $x_{1}=0$ (this is possible due to the upper triangular structure of $A_{\mathrm{cl}}$ and $E_{\mathrm{cl}}$ ). Then we observe that this projection of $\Gamma_{2}$ is forward invariant and globally uniformly attractive (because the persistently flowing solutions from $\Gamma_{3}$ converge to zero after the first jump in at most $\tau_{\max }$ ordinary time). Using the fact that the projection of $\Gamma_{2}$ on the $x_{1}=0$ hyperplane is compact, GAS of $\Gamma_{2}$ relative to $\Gamma_{3}$ follows from ${ }^{1}$ [17, Prop. 7.5].

2) All solutions are bounded. Indeed, pick an arbitrary initial condition and note that $a$ ) state $x_{5}$ is bounded because it does not change across jumps and converges to zero following a linear exponentially convergent transient during flows; $b$ ) state $\left(x_{3}, x_{4}\right)$ follows analogous dynamics, also being perturbed by a bounded input during flows, which cannot drive the state unbounded from bounded input bounded state stability properties of linear exponentially stable continuous-time dynamics; c) boundedness of $x_{2}$ follows from the fact that it remains constant along flows and jumps to some linear combinations of bounded quantities $\left(x_{3}, x_{4}, x_{5}\right)$ across jumps; $d$ ) state $x_{1}$ is then bounded by a straightforward application of [20, Lemma 1], because the linear time-invariant dynamics is governed by an exponentially stable linear time-invariant dynamics along (persistent) flows (see the assumption on $A+B K_{1}$ in (13)) and by the identity map across jumps, whereas the forcing inputs acting along flows and across jumps come as linear combinations of bounded variables $\left(x_{1}, x_{2}, x_{3}\right)$.

The above properties 1) and 2) imply that we can invoke [19, Thm 4] and global asymptotic stability of $\mathcal{A}$ follows.

Proof of item 2). We start proving that there exists a $q$ dependent matrix $\Pi(q) \in \mathbb{R}^{8 \times 1}$ that satisfies the following conditions

$$
\begin{array}{r}
{\left[\begin{array}{cc}
A_{\mathrm{cl}}(q) & G_{\mathrm{cl}}(q) \\
C_{\mathrm{cl}}(q) & D_{\mathrm{cl}}
\end{array}\right]\left[\begin{array}{c}
\Pi(q) \\
I
\end{array}\right]=0, \quad \forall q \in \mathcal{Q}} \\
\Pi\left(q^{+}\right)-E_{\mathrm{cl}}(q) \Pi(q)=0, \quad \forall q \in \mathcal{Q},
\end{array}
$$

\footnotetext{
${ }^{1}$ The statement in [17, Prop. 7.5] is only local but a global version of it trivially follows from picking increasingly large values of the scalar $\mu$ therein characterized.
} 


$\left[\begin{array}{c|c}A_{\mathrm{cl}}(q) & G_{\mathrm{cl}}(q) \\ \hline E_{\mathrm{cl}}(q) & \\ \hline C_{\mathrm{cl}}(q) & D_{\mathrm{cl}}(q)\end{array}\right]:=\left[\begin{array}{ccccc|c}A+B K_{1} & (2-q) B & 0 & 0 & (2-q) B K_{1} & (2-q) G \\ 0 & 0 & 0 & 0 & 0 & 0 \\ 0 & 0 & A+B K_{2} & B K_{\mathrm{i}} & B K_{2} & G \\ 0 & 0 & C & 0 & 0 & Q \\ 0 & 0 & 0 & 0 & A+L C & -G-L Q \\ \hline I & 0 & (2 q-3) I & 0 & 0 & \\ 0 & 0 & K_{2}-K_{1} & K_{\mathrm{i}} & K_{2}-K_{1} & \\ 0 & 0 & I & 0 & 0 & \\ 0 & 0 & 0 & I & 0 & \\ 0 & 0 & 0 & 0 & I & \\ \hline C & 0 & (q-1) C & 0 & 0 & Q\end{array}\right]$

which are a generalization of the classical regulator equations [21]. The conditions in (16) can be intuitively derived noticing that $x(\infty):=\Pi(q) w(\infty)$, and $e(\infty):=C_{\mathrm{cl}}(q) x(\infty)+$ $D_{\mathrm{cl}} w(\infty)$, so that the flow properties $(\dot{x}(\infty), e(\infty))=(0,0)$ can be derived by imposing $\Pi\left(q^{+}\right) w(\infty)=x(\infty)^{+}=$ $E_{\mathrm{cl}}(q) x(\infty)$, and where we used the notation $(\infty)$ to denote the asymptotic value.

In order to prove (16) we split the subspace $\Pi(q)$ according to the partitioning in (14) as follows $\Pi(q):=$ $\left(\Pi_{1}(q), \Pi_{2}, \Pi_{3}, \Pi_{4}, \Pi_{5}\right)$, and considering (16a) we obtain the following set of equalities

$$
\begin{aligned}
& 0=\left(A+B K_{1}\right) \Pi_{1}(q)+(2-q)\left(B \Pi_{2}+B K_{1} \Pi_{5}+G\right) \\
& 0=\left(A+B K_{2}\right) \Pi_{3}+B K_{\mathrm{i}} \Pi_{4}+B K_{2} \Pi_{5}+G \\
& 0=C \Pi_{3}+Q \\
& 0=(A+L C) \Pi_{5}-G-L Q
\end{aligned}
$$

where we omitted equations that are trivially satisfied. By the internal model principle [21] we know that (17b), (17c) and (17d) are automatically satisfied. Now, we can easily verify that, with the selection

$$
\begin{aligned}
\Pi_{1}(q) & =(2-q) \Pi_{3} \\
\Pi_{2} & =K_{\mathrm{i}} \Pi_{4}+\left(K_{2}-K_{1}\right)\left(\Pi_{3}+\Pi_{5}\right),
\end{aligned}
$$

equation (17a) reduces to (17b) and it is automatically satisfied by the internal model principle. It is not hard to check that with the selection (18) also (16b) is satisfied. Finally, plugging the change of coordinates $\tilde{x}=x-\Pi(q) w$ into (15), and using (16), we obtain a hybrid system equivalent to (15) where $w$ has been set to zero and whose stability has already been proved, which concludes the proof.

\section{Controller tuning}

In this section we propose an LMI-based technique to tune the controller parameters $K_{1}, K_{2}, K_{\mathrm{i}}$, and $L$. We remark that, due to the special cascaded structure of (11), all these parameters can be tuned independently.

\section{A. Transient mode tuning}

We propose to choose $K_{1}$ following a two-step procedure generalizing the results of [8]. As a first step we characterize the maximum achievable convergence rate $\alpha \in \mathbb{R}_{>0}$ by imposing constraints on the aggressiveness of the feedback gain $K_{1}$ (a bound on its norm) and on the closed-loop damping factor. Specifically, we aim to solve the following optimization problem:

$$
\begin{aligned}
& \max _{W, X, \alpha} \alpha \quad \text { subject to: } \\
& W=W^{\top} \geq I \\
& M+M^{\top} \leq-2 \alpha W \\
& {\left[\begin{array}{ll}
\left(M+M^{\top}\right) \sin \theta \quad\left(M-M^{\top}\right) \cos \theta \\
\left(M^{\top}-M\right) \cos \theta \quad\left(M+M^{\top}\right) \sin \theta
\end{array}\right] \leq 0} \\
& {\left[\begin{array}{ll}
W & X^{\top} \\
X & I \kappa^{2}
\end{array}\right] \geq 0,}
\end{aligned}
$$

where $M:=A W+B X \in \mathbb{R}^{2 \times 2}$ and $\theta \in[0, \pi / 2]$. We used the following notation: given a matrix $M, M^{\top}$ denotes its transpose and, for square matrices, $M>0(M \geq 0)$ denotes positive definiteness (semi-definiteness). The identity matrix of proper dimensions is denoted by $I$.

Optimization problem (19) is non convex due to the product $\alpha W$, but it can be efficiently solved because it is a quasiconvex generalized eigenvalue problem [22]. The next proposition establishes a few useful properties for (19).

Proposition 1. Under Assumption 2, for any value of $\kappa \in$ $\mathbb{R}_{>0}$, there exists a large enough $\theta \in[0, \pi / 2]$ such that (19) is feasible. Moreover, for any feasible solution to (19) together with the choice $K_{1}=X W^{-1}$, the following properties hold: i) the norm $\left|K_{1}\right| \leq \kappa$, ii) the closed-loop matrix $\left(A+B K_{1}\right)$ has eigenvalues with real part less than $-\alpha$, iii) the damping factor of the poles is larger than $\cos \theta$.

Proof. Feasibility follows from the fact that, with $\theta=\pi / 2$, constraint (19c) reduces to $M+M^{\top} \leq 0$. Then the assumption that $A$ be Hurwitz implies that $X=0$ is a feasible solution for any non-negative value of $\kappa$. To show that $\left|K_{1}\right| \leq \kappa$, a Schur complement on (19d) and $X=K_{1} W$ and (19a) yield

$$
I \kappa^{2} \geq X W^{-1} X^{\top}=K_{1} W K_{1}^{\top} \geq K_{1} K_{1}^{\top},
$$

which implies $\left|K_{1}\right| \leq \kappa$. The eigenvalues of $\left(A+B K_{1}\right)$ having real part less than $-\alpha$ is a straightforward consequence of (19b), and the damping factor greater than $\cos \theta$ is a direct application of the results in [23, Equation (13)].

The optimization problem (19) provides a convenient way to trade-off between speed of convergence, imposed by $\alpha$, and level of aggressiveness of the arising controller, tuned by $\kappa$.

The second step of the proposed synthesis procedure consists in reducing the overshoot associated to the gain selection 


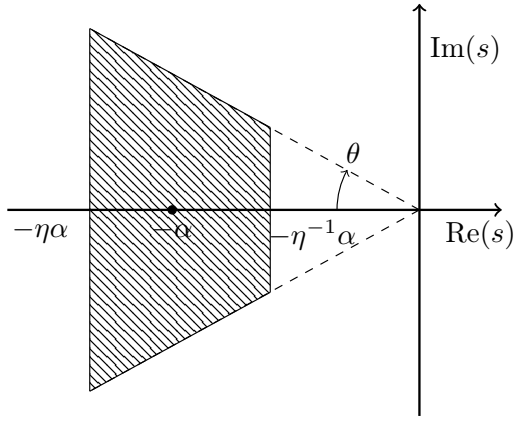

Fig. 6. The shaded conic region represents the region in the complex plane where the closed-loop poles are constrained.

obtained with (19). Toward this goal we impose the nonovershooting condition by forcing a small constant of proportionality between the derivative of the tracking error $\dot{e}$ and the partial state $x_{1}$, when $e=0$. We formalize this idea by imposing the following conservative bound

$$
e=0 \quad \Rightarrow \quad|\dot{e}| \leq \rho\left|x_{1}\right|,
$$

and we minimize $\rho \in \mathbb{R}_{>0}$. When constant $\rho$ is zero, a zero overshoot is ensured by the forward invariance of the zero error manifold, (in other words overshoot is impossible with $\rho=0$ because crossing the set where $e=0$ contradicts (20)), but in cases where the absence of overshoot cannot be attained due to intrinsic limitations, a minimized overshoot is conveniently obtained. Since $x_{1}$ is the only non-zero element of the state $x$ whenever $e=0$, Equation (20) can be equivalently re-written in the following way

$$
\left|C\left(A+B K_{1}\right) x_{1}\right| \leq \rho\left|x_{1}\right|, \quad \forall x_{1}: C x_{1}=0 .
$$

An LMI formulation of (21) is included in the next optimization problem minimizing the overshoot metric $\rho$.

$$
\begin{aligned}
& \min _{W, S, X, \rho^{2}} \rho^{2} \quad \text { subject to: } \\
& M+M^{\top} \leq-\eta^{-1} \alpha W \\
& M+M^{\top} \geq-\eta \alpha W \\
& {\left[\begin{array}{ccc}
2 W & M C^{\top} & I \\
C M & \rho^{2} I & 0 \\
I & 0 & I+C^{\top} S C
\end{array}\right]>0} \\
& S=S^{\top}>0
\end{aligned}
$$

$$
(19 a),(19 c),(19 d) \text {. }
$$

Remark 5. Constraints (22a), (22b) and (19c) force the closedloop poles to lie in the shaded gray region of the complex plane shown in Figure 6. The shape of this region can be adjusted using parameters $\eta$ and $\alpha$.

Proposition 2. Under Assumption 2, assume that $\alpha, \theta, \kappa$ are parameters for which (19) is feasible; then there exists a large enough $\eta \in \mathbb{R}_{\geq 1}$ such that (22) is feasible as well.

Moreover, for any feasible solution to (22), selecting $K_{1}=$ $X W^{-1}$ the following properties hold: $\left.i\right)$ the norm $\left|K_{1}\right| \leq \kappa$, ii) the closed-loop matrix $\left(A+B K_{1}\right)$ has eigenvalues with real part less than $-\alpha \eta^{-1}$, iii) the damping factor of the poles is greater than $\cos \theta, i v)$ the relation in (20) holds.

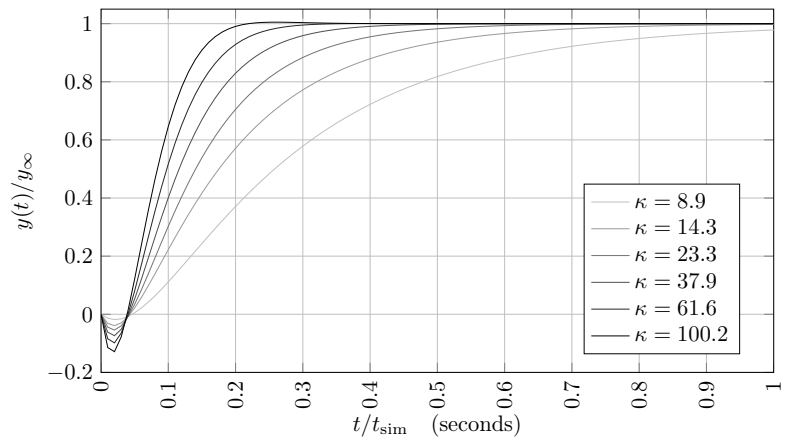

Fig. 7. Simulated step responses for increasing values of $\kappa$. The time scale is normalized.

Proof. Feasibility holds because there exists $\eta$ sufficiently large such that the shaded region in Figure 6 contains the positions of the poles obtained by the feasible solution for (19), assumed in Proposition 2. Then that solution is also feasible for (22) as long as $\rho^{2}$ is sufficiently large. Properties i)-iii) are a straightforward consequence of constraints (19a), (19c), (19d), which imply the stated properties from Proposition 1. We finish the proof by showing property (4). Performing a Schur complement on (22c), and using (22d), we obtain:

$$
\left[\begin{array}{cc}
2 W-\left(I+C^{\top} S C\right)^{-1} & M^{\top} C^{\top} \\
C M & \rho^{2} I
\end{array}\right]>0
$$

where $I+C^{\top} S C$ is clearly invertible. Consider now the following inequality

$\left(W-\left(I+C^{\top} S C\right)^{-1}\right)^{\top}\left(I+C^{\top} S C\right)\left(W-\left(I+C^{\top} S C\right)^{-1}\right) \geq 0$, which implies $2 W-\left(I+C^{\top} S C\right)^{-1} \leq W\left(I+C^{\top} S C\right) W$. From (23), pre/post multiplying by $\operatorname{diag}\left(W^{-1}, I\right)$ and using $M=\left(A+B K_{1}\right) W$, we obtain

$$
\left[\begin{array}{cc}
I+C^{\top} S C & \left(A+B K_{1}\right)^{\top} C^{\top} \\
C\left(A+B K_{1}\right) & \rho^{2} I
\end{array}\right]>0
$$

which, after being multiplied by $\rho^{2}$ and after a Schur complement, implies:

$$
\left|C\left(A+B K_{1}\right) x_{1}\right|^{2}<\rho^{2}\left|x_{1}\right|^{2}+\rho^{2}\left|\sqrt{S} C x_{1}\right|^{2} .
$$

Finally, when $C x_{1}=0$, a square root gives (21).

The properties established in Propositions 1 and 2 suggest that the feedback gain $K_{1}$ be selected as follows: first a set of different levels of aggressiveness are fixed, spanning an experimentally reasonable range. Then for each one of them we solve the optimization problem in (19), possibly adjusting the parameter $\theta$ if the LMIs are infeasible (feasibility is guaranteed by Proposition 1 for a large enough $\theta$ ). This first step provides a number of values for $\alpha$, each of them corresponding to a different selection of $\kappa$. As a second step, optimization (22) is solved for each one of the $(\kappa, \alpha)$ pairs with the same value of $\theta$, possibly adjusting parameter $\eta$ (feasibility is guaranteed by Proposition 2 for a large enough $\eta$ ).

For the model identified in this work, the values of $\kappa$ are shown in Figure 7 with the resulting step responses, confirming the non-overshooting feature ensured by Proposition 1. The corresponding values for $\alpha$ have been obtained by applying optimization (19) after fixing $\theta=\pi / 4$ and $\eta=5$. 


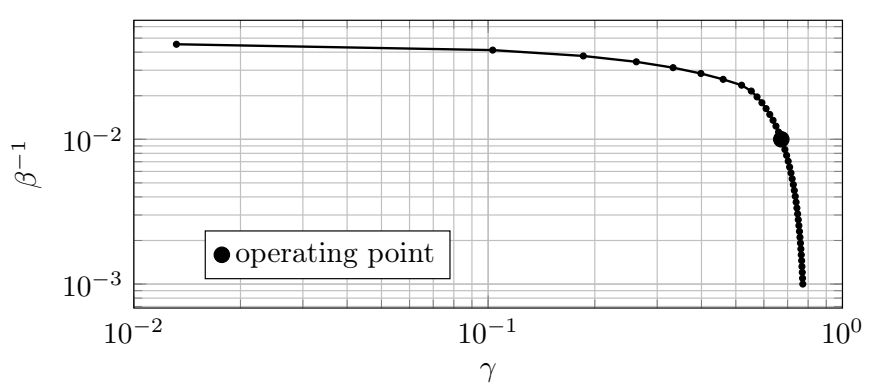

Fig. 8. Trade-off curve between $\beta$ and $\gamma$. Curves obtained by solving the optimization problem (25) for model (4), considering increasing values of $\beta$.

\section{B. Steady-state mode tuning}

In this section we provide the details of the synthesis of the controller gains $K_{2}$ and $K_{\mathrm{i}}$. In practice we tuned these gains, associated with the dynamics of the second matrix in (13), in such a way that its convergence rate is larger than the convergence rate $\alpha$ obtained by the optimization problem (22). In this way, when switching from the transient mode to the steady-state mode, the integral action associated to state $x_{\mathrm{c} 4}$ is already almost constant. We also remark that, for the steadystate dynamics, no special non-overshooting requirements are imposed. Indeed, from (5) we can observe that these gains have no effect during the transient mode. Consequently, the convergence rate of this dynamics can be made arbitrarily large without affecting the non-overshooting features ensured by the transient mode controller.

\section{Observer design}

In this section we tune the observer gain $L$ as a convenient trade-off between convergence rate of the estimation error and noise rejection capability. For the estimation error dynamics

$$
\dot{x}_{5}=(A+L C) x_{5}-G w-L Q w,
$$

which emerges from (14), we propose to optimize the observer gain $L$ in order to reduce the effect of disturbance $w$ on the estimation error in the $\mathcal{L}_{2}$ sense. Following the well-known Lyapunov formulations of the bounded real lemma (see, e.g, [22]), we select $L$ according to the following LMI-based convex optimization problem, parametrized by $\beta \in \mathbb{R}_{>0}$,

$$
\begin{aligned}
& \min _{P, Y, \gamma} \gamma \text { subject to: } \\
& P=P^{\top}>0 \\
& \mathrm{He}\left[\begin{array}{ccc}
P A+Y C & -Y Q-P G & 0 \\
0 & -\gamma I / 2 & 0 \\
I & 0 & -\gamma I / 2
\end{array}\right]<0 \\
& \mathrm{He}[P A+Y C] \leq-2 \beta P,
\end{aligned}
$$

with the shortcut notation $\operatorname{He}(M):=M+M^{\top}$.

Proposition 3. Given any $\alpha \in \mathbb{R}_{>0}$, LMI (25) is feasible under Assumption 2. Moreover, for any feasible solution to (25), selecting $L:=P^{-1} Y$ it holds that: $\left.i\right)$ the $\mathcal{L}_{2}$ gain from $w$ to $x_{5}$ for (24) is smaller than $\gamma$, ii) matrix $A+L C$ has eigenvalues with real part smaller than or equal to $-\beta$.
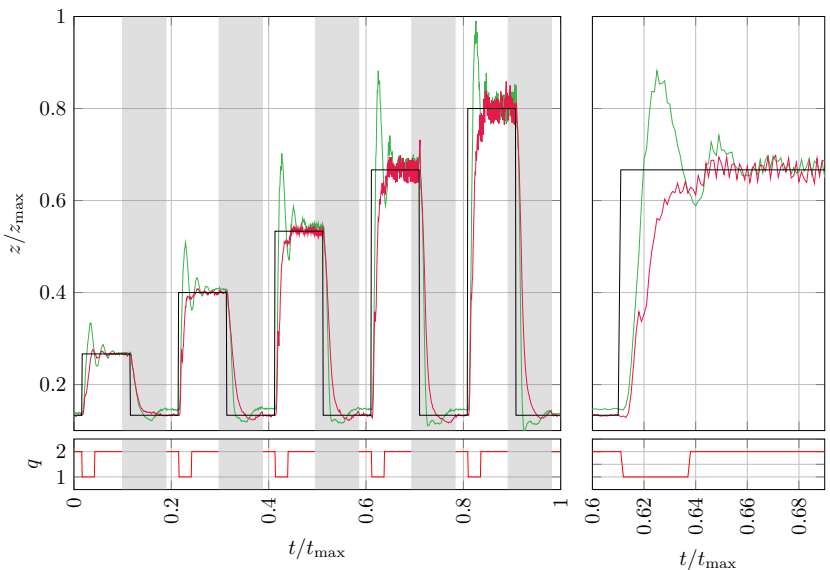

Fig. 9. Open-loop vs. closed-loop step response. In green open-loop, in red closed-loop and in black the reference. On the left a sequence of increasing amplitude steps and on the right a zoom of the fourth step.

Proof. Feasibility of (25c) follows from standard pole placement theory combined with minimality, see Assumption 2. The remaining part of the proof is a standard application of the bounded real lemma and the use of quadratic Lyapunov functions. In particular, defining $V\left(x_{5}\right):=x_{5}^{\top} P x_{5}$, where $P=P^{\top}>0$ by constraint (25a), performing a Schur complement on $(25 \mathrm{~b})$, left-right-multiplying by $\left(x_{5}, w\right)$ and substituting $Y=P L$, we obtain $\left\langle\nabla V\left(x_{5}\right), \dot{x}_{5}\right\rangle+\frac{1}{\gamma} x_{5}^{\top} x_{5}-$ $\gamma w^{\top} w<0$.

Integrating both sides, we obtain the desired bound on the $\mathcal{L}_{2}$ gain from $w$ to $x_{5}$ (or equivalently on the $\mathcal{H}_{\infty}$ norm). Regarding the speed of convergence $\beta$, this follows from noticing that (25c) implies $\mathrm{He}[P(A+L C+\beta I)]<0$, which only holds with a positive definite $P$ if $A+L C$ has convergence abscissa smaller than $-\beta$.

Proposition 3 emphasizes that the LMI-based design tool corresponding to (25) can be an effective means for performing the design of $L$, while establishing a trade-off between the guaranteed speed of convergence of the observer dynamics (corresponding to $\beta$ ) and the level of disturbance rejection $\gamma$ from the noise $w$ to the estimation error $x_{5}$. The suggested use of this tool is to fix increasing values of $\beta$ and then determine the trade-off curve reported in Figure 8. This curve provides a range of optimal selections of the observer gain $L$. For our specific identified model, Figure 8 reports the selected operating point, corresponding to a black dot. Such a selection is performed by fixing a sufficiently large convergence rate $\beta$ once the state feedback gains have been designed.

\section{EXPERIMENTAL VALIDATION}

All the experiments have been conducted on the testing facilities provided by DRTS. The hybrid controller (8) has been implemented on a Bosch ${ }^{\circledR}$ electronic control unit for rapid prototyping. The code has been generated directly from TargetLink ${ }^{\circledR}$ by discretizing the controller with the Tustin method with a sampling time of $2 \mathrm{~ms}$ (this is indeed possible because the controller is linear along the flow). The reset action has been coded using the external reset signal readily available in the Simulink integrator block. Tests have been conducted 


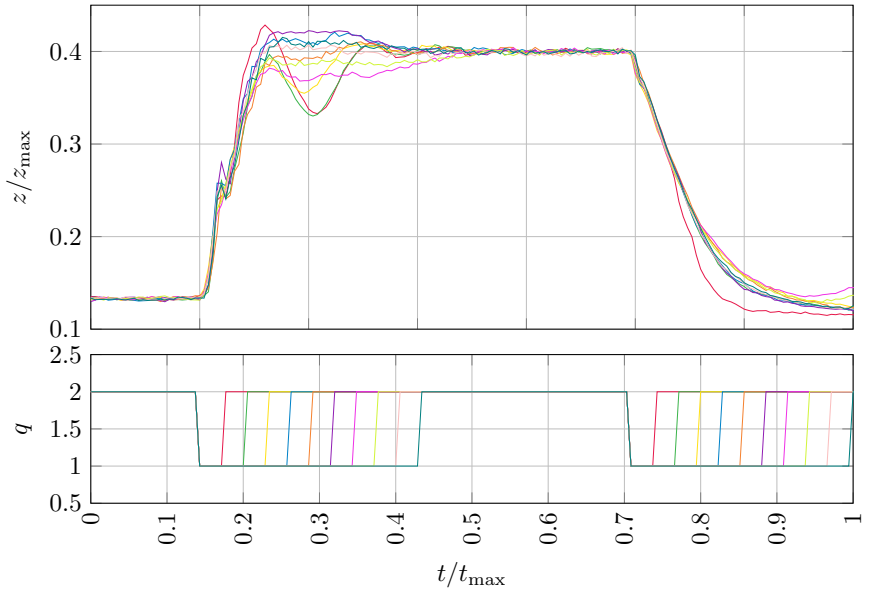

Fig. 10. Step responses for different $t_{\text {switch }}$. In the graph above the pressure, below the logic variable $q$.

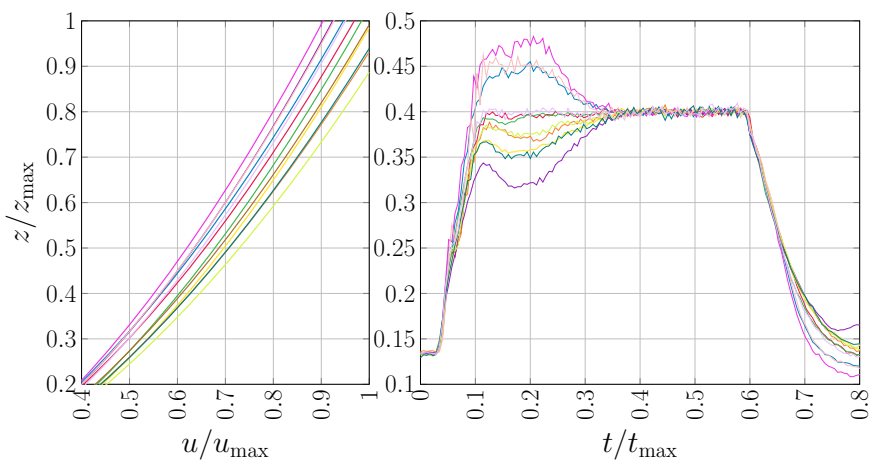

Fig. 11. Step responses (right) with variations of the model $\phi$ (left).

over a range of five different clutches that differ in dimensions, transmissible torque, stiffness and hydraulic properties. For each one of these clutches, we performed the identification procedure described in Section III and the controller tuning procedure reported in Section V. Several experimental tests have been performed, providing excellent results for a large variety of working conditions. Figure 9 shows the comparison between the closed-loop (in red), and the open loop (in green). The controller successfully removes the overshoot and ensures a zero steady state error. The switch among controller 1 and 2 is ruled by the logic variable $q$ reported in Figure 9 as well.

The value of $q$ toggles according to a timer that is reset to zero at each rising/falling of the reference signal. Therefore after a time $t_{\text {switch }} \in \mathbb{R}_{>0}$ from the last reference change, the variable $q$ toggles from 1 to 2 and the controller switches from the transient mode to the steady state one. The time $t_{\text {switch }}$ can be easily calibrated experimentally through a bisection procedure. In Figure 10 we show the set of responses obtained by varying $t_{\text {switch }}$ between 0 and the settling time of the closed-loop. We can observe that, for a reasonable range of values around the optimum, the closed-loop response remains non-overshooting, so that the feature ensured by the transient mode controller is successfully attained on the experimental plant also in the presence of imprecise switching. It is also emphasized that the variable $q$ toggles according to a timer, but in principle more complicated strategies are possible, thanks to the stability properties proved in Theorem 1 .

Finally, we tested the robustness of the proposed controller against large perturbations of the model of the nonlinear map $\phi$, possibly caused by oil aging and temperature variations. Those perturbations are reported on the left in Figure 11, while on the right the corresponding step responses are reported. We notice that for very large variations of $\phi$, the non-overshooting property is not preserved, but the integral action is still able to zero out the steady state error. Those perturbation are large as compared to the ones experienced in practice (roughly three times larger), and the degradation of performance is graceful. This shows a desirable level of robustness.

We conclude that the experimental tests have proved the validity of the proposed control technique for achieving a non-overshooting and fast pressure response. Moreover, we experimentally tested robustness with respect to perturbations of the nonlinear map $\phi$ and of the switching time $t_{\text {switch }}$.

\section{CONCLUSIONS}

In this paper developed a control-oriented model for a filled clutch in the modulation range and we proposed a novel hybrid controller. The controller can operate in two modes, a transient mode and a steady-state mode, and may freely switch among these modes without compromising stability. The transient mode provides a nice non-overshooting response, while the steady-state mode compensates for constant disturbances and unmodeled dynamics. The controller synthesis is conveniently formulated as an LMI problem. We implemented and tested the proposed hybrid controller on the HVT developed by DRTS. Experiments show excellent performance and good robustness with respect to modeling errors and noise. Future directions include the design of high-level switching strategies and oil pressure control in partially filled clutches.

\section{REFERENCES}

[1] M. Mueller, W. Scandella, and S. Mutschler, "Power-split transmission for a traction drive and method for controlling the transmission," Jan. 26 2016, uS Patent 9,243,701. [Online]. Available: https: //www.google.ch/patents/US9243701

[2] J. Horn, J. Bamberger, P. Michau, and S. Pindl, "Flatness-based clutch control for automated manual transmissions," Control Engineering Practice, vol. 11, no. 12, pp. 1353-1359, 2003.

[3] H. Hao, T. Lu, J. Zhang, and B. Zhou, "A new control strategy of the filling phase for wet dual clutch transmission," Proceedings of the Institution of Mechanical Engineers, Part C: Journal of Mechanical Engineering Science, p. 0954406215590187, 2015.

[4] X. Song and Z. Sun, "Pressure-based clutch control for automotive transmissions using a sliding-mode controller," Mechatronics, IEEE/ASME Transactions on, vol. 17, no. 3, pp. 534-546, 2012.

[5] M. Goetz, M. Levesley, and D. Crolla, "Dynamics and control of gearshifts on twin-clutch transmissions," Proceedings of the institution of mechanical engineers, Part D: Journal of Automobile Engineering, vol. 219, no. 8, pp. 951-963, 2005.

[6] O. Beker, C. Hollot, and Y. Chait, "Plant with an integrator: an example of reset control overcoming limitations of linear feedback," IEEE Transactions Automatic Control, vol. 46, pp. 1797-1799, 2001.

[7] M. M. Seron, J. H. Braslavsky, and G. C. Goodwin, Fundamental limitations in filtering and control. Springer Science \& Business Media, 2012.

[8] L. De Pascali, M. Cocetti, F. Biral, A. Palazzetti, F. Panizzolo, P. Rinaldi, A. Sassaro, and L. Zaccarian, "Lmi-based non-overshooting pressure control design for a wet clutch," in Control Applications (CCA), 2016 IEEE Conference on. IEEE, 2016, pp. 329-334. 
[9] L. Eriksson and L. Nielsen, Modeling and control of engines and drivelines. John Wiley \& Sons, 2014.

[10] M. Cordioli, M. Mueller, F. Panizzolo, F. Biral, and L. Zaccarian, "An adaptive reset control scheme for valve current tracking in a power-split transmission system," in Control Conference (ECC), 2015 European. IEEE, 2015, pp. 1884-1889.

[11] L. Ljung, "System identification: Theory for the user, ptr prentice hall information and system sciences series," 1999.

[12] B. Moore, "Principal component analysis in linear systems: Controllability, observability, and model reduction," IEEE transactions on automatic control, vol. 26, no. 1, pp. 17-32, 1981.

[13] A. S. Morse, "Supervisory control of families of linear set-point controllers. 2. robustness," IEEE Transactions on Automatic Control, vol. 42, no. 11, pp. 1500-1515, Nov 1997.

[14] J. P. Hespanha and A. Morse, "Switching between stabilizing controllers," Automatica, vol. 38, no. 11, pp. 1905 - 1917, 2002.

[15] D. Liberzon, J. ao P. Hespanha, and A. Morse, "Stability of switched systems: a lie-algebraic condition," Systems and Control Letters, vol. 37, no. 3, pp. $117-122,1999$.

[16] L. Zaccarian and A. R. Teel, "The $\mathcal{L}_{2}\left(\ell_{2}\right)$ bumpless transfer problem for linear plants: Its definition and solution," Automatica, vol. 41, no. 7, pp. 1273-1280, 2005

[17] R. Goebel, R. G. Sanfelice, and A. R. Teel, "Hybrid dynamical systems: modeling, stability, and robustness," 2012.

[18] C. Cai, A. R. Teel, and R. Goebel, "Smooth lyapunov functions for hybrid systems part ii: (pre)asymptotically stable compact sets," IEEE Transactions on Automatic Control, vol. 53, no. 3, pp. 734-748, April 2008.

[19] M. Maggiore, M. Sassano, and L. Zaccarian, "Reduction theorems for hybrid dynamical systems," IEEE Transactions on Automatic Control, to appear, 2018.

[20] N. Noroozi, D. Nešić, and A. R. Teel, "Gronwall inequality for hybrid systems," Automatica, vol. 50, no. 10, pp. 2718 - 2722, 2014.

[21] B. A. Francis, "The linear multivariable regulator problem," SIAM Journal on Control and Optimization, vol. 15, no. 3, pp. 486-505, 1977.

[22] S. Boyd, L. E. Ghaoui, E. Feron, and V. Balakrishnan, Linear Matrix Inequalities in System and Control Theory. Society for Industrial an Applied Mathematics, 1994.

[23] M. Chilali and P. Gahinet, " $\mathcal{H}_{\infty}$ design with pole placement constraints: an LMI approach," IEEE Transactions on Automatic Control, vol. 41, no. 3, pp. 358-367, 1996.

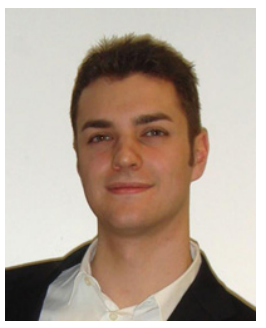

Matteo Cocetti received the B.Eng. in Mechatronic from the University of Modena e Reggio Emilia, IT, and the M. Eng. in Robotics from the University of Trento, IT, both "cum laude". During his studies has been visiting student at The Ohio State University, Columbus, $\mathrm{OH}$. He is currently enrolled in a joint $\mathrm{Ph}$.D. program between the University of Trento and the National Institute of Applied Sciences INSA, FR. His research interests include output regulation for over-actuated systems, hybrid systems and distributed control of robots.

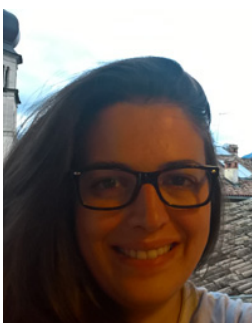

Silvia Donnarumma was born in Genoa in 1986 She received the Master Degree in Naval Architecture and Marine Engineering and the Ph.D. in Mathematical Engineering and Simulation, both at the University of Genoa. After the Ph.D. she got research fellowships at the University of Genoa, and at the University of Trento. She is currently a postdoctoral researcher at the University of Genoa. Her main interest include automatic steering/ positioning systems, LMI-based control, convex optimization, and control of systems with saturation.

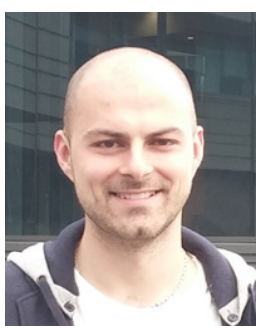

Luca De Pascali received the M.Sc. in Mechatronic Engineering in 2015 from the University of Trento (Italy). During his studies has been visiting student at Technische Universität München (Germany). In 2016 he was visiting scholar at Clemson University - International Center for Automotive Research (USA). He is currently enrolled as a Ph.D. candidate at the Department of Industrial Engineering of the University of Trento. His research interests include optimal control of hybrid dynamical systems and hybrid power-train modeling and control.

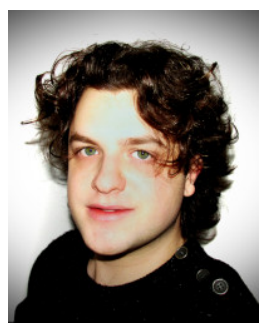

Matteo Ragni was born in Italy, on December 2nd, 1989. He received the Ph.D. in Materials, Mechatronic and Systems Engineering from the University of Trento, (IT). He is currently a post-doctoral researcher at the University of Trento, (IT). His research interests are machine learning, intelligent manufacturing systems, and augmented reality. $\mathrm{He}$ is currently working on high-performance control for wet-clutches for off-highway vehicles.

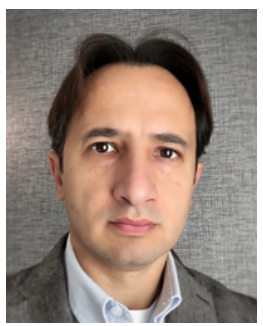

Francesco Biral received the Master Degree in Mechanical Engineering at the University of Padova, Italy, and the Ph.D. in Mechanism and Machine Theory from University of Brescia, Italy, in 2000 . He is currently Associate Professor at the Department of Industrial Engineering at University of Trento. His research interests include symbolic and numerical multi-body dynamics and optimization, constrained optimal control, mainly in the field of vehicle dynamics with special focus on Intelligent Transportation Systems.



Fabrizio Panizzolo received his degree in Mechanical Engineering from the University of Padua in 1980. He has been Senior Engineer at Dana OffHighway and he is currently the technical director for Dana Rexroth Transmission Systems. He is the author of more than twenty patents related to off-highway drive train components. His research interests are mechatronic and the design of highperformance power-split transmissions.

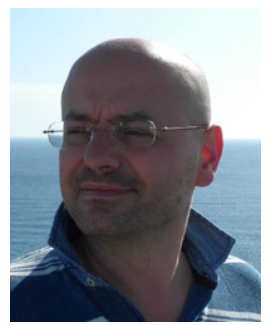

Pier Paolo Rinaldi received the Laurea Degree in Mechanical Engineering from Polytechnic of Turin and a M.Sc. in Automotive Engineering from University of Modena and Reggio Emilia. During his studies he has been visiting student at the University of Sheffield. He is currently collaborating with the Human Centred Design Institute, Polytechnic University of Milan, and with the University of Bologna, on topics related to vibration mechanics. Throughout his career, mainly in high-end sports cars and offhighway industry, he has been working on vibration mechanics and design of high-performance transmissions systems.

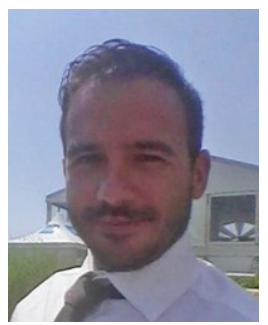

Alex Sassaro received the B.Eng. and the M.Eng. degree in Automation from the University of Padua, Italy. From 2010 to 2011, he was a member of the R\&D team at DANA Italia, Arco, Italy, working on the development and testing of off-highway transmissions systems. From 2011 to 2013 he has been working with the software team at Dana-Rexroth Transmission Systems, developing hardware in the loop simulations and automatic tests for automotive applications. He currently works on the HVT project developing clutch control algorithms. His main interests are model-based design and real-time control of transmissions systems.

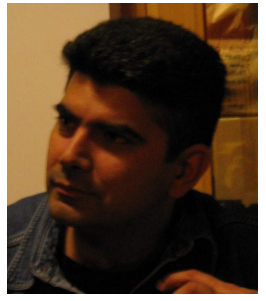

Luca Zaccarian (SM '09 - F' 16) received the Laurea and the Ph.D. degrees from the University of Roma Tor Vergata (Italy) where has been Assistant Professor in control engineering from 2000 to 2006 and then Associate Professor. Since 2011 he is Directeur de Recherche at the LAAS-CNRS, Toulouse (France) and since 2013 he holds a parttime associate professor position at the University of Trento, Italy. Luca Zaccarian's main research interests include nonlinear and hybrid control systems, modeling and control of mechatronic systems. 